\title{
CRITERION FOR EXISTENCE OF A LOGARITHMIC CONNECTION ON A PRINCIPAL BUNDLE OVER A SMOOTH COMPLEX PROJECTIVE VARIETY
}

\author{
SUDARSHAN GURJAR AND ARJUN PAUL
}

\begin{abstract}
Let $X$ be a connected smooth complex projective variety of dimension $n \geq 1$. Let $D$ be a simple normal crossing divisor on $X$. Let $G$ be a connected complex Lie group, and $E_{G}$ a holomorphic principal $G$-bundle on $X$. In this article, we give criterion for existence of a logarithmic connection on $E_{G}$ singular along $D$.
\end{abstract}

\section{INTRODUCTION}

A theorem of Weil [Wei38] says that a holomorphic vector bundle $E$ on a smooth complex projective curve $X$ admits a holomorphic connection if and only if each indecomposable holomorphic direct summand of $E$ has degree 0; see [Ati57]. For connected reductive linear algebraic group $G$ over $\mathbb{C}$, this result of Weil and Atiyah is generalized to the case of holomorphic principal $G$-bundles on a smooth complex projective curve in [AB02]. It follows from [Ati57, Theorem 4, p. 192] that, not every holomorphic bundle on a compact Kähler manifold can admit a holomorphic connection. Therefore, one can ask for criterion for a holomorphic bundle on $X$ to admit a meromorphic connection. Simplest case of meromorphic connection is logarithmic connection. So it natural to ask when a given holomorphic bundle on $X$ admits a logarithmic connection singular along a given divisor with prescribed residues. When $X$ is a smooth complex projective curve, in [BDP18], a necessary and sufficient criterion for a vector bundle on $X$ to admit a logarithmic connection singular along a given reduced effective divisor $D$ on $X$ with prescribed rigid residues along $D$ is given. This result is further generalized to the case of holomorphic principal $G$-bundles over smooth complex projective curve in [BDPS17] when $G$ is a connected reductive linear algebraic group over $\mathbb{C}$. When $X$ is a smooth complex projective variety of dimension of more than one, no such criterion for existence of logarithmic connection on a holomorphic bundle on $X$ with prescribed residues along a given reduced effective divisor is known to the best of our knowledge. In this article, we attempt to study this problem.

Date: July 1, 2020.

2010 Mathematics Subject Classification. 14J60, 53C07, 32L10.

Key words and phrases. Logarithmic connection; residue; vector bundle; principal bundle.

Corresponding author: Arjun Paul. 
1.1. Outline of the paper. Unless otherwise specified, $X$ is a connected smooth complex projective variety of dimension at least one, and $D$ a reduced effective divisor on $X$. We denote by $G$ a connected affine algebraic group over $\mathbb{C}$. In Section $\S 2$, we recall definitions of simple normal crossing divisor, logarithmic connection on holomorphic vector bundles on $X$, and their residues along a simple normal crossing divisor on $X$. In Section $\S 3$, we extend this notion of logarithmic connection for principal $G$-bundles $E_{G}$ on $X$, and discuss the notion of residue of a logarithmic connection on $E_{G}$ singular along a simple normal crossing divisor on $X$.

In Section $§ 3.3$, we study logarithmic connection on principal bundles under the extensions of structure group. Let $H$ be a connected closed algebraic subgroup of $G$ over $\mathbb{C}$. Let $E_{H}$ be a holomorphic principal $H$-bundle on $X$. Let $E_{H}(G)$ be the holomorphic principal $G$ bundle on $X$ obtained by extending the structure group of $E_{H}$ by the inclusion map $H \subset G$. Then we have the following (see Proposition 3.3.1).

Proposition 1.1.1. If $E_{H}$ admits a logarithmic connection singular along $D$, then $E_{H}(G)$ admits a logarithmic connection singular along $D$. The converse holds if $H$ is reductive.

The case of parabolic subgroup $P$ of a reductive affine algebraic group $G$ over $\mathbb{C}$ is interesting. Let $L \cong P / R_{u}(P)$ be the Levi factor of $P$, where $R_{u}(P)$ is the unipotent radical of $P$. Let $E_{L}$ be the corresponding holomorphic principal $L$-bundle on $X$ obtained by extending the structure group from $P$ to $L$. The natural action of $P$ on the Lie algebra $\mathfrak{n}:=\operatorname{Lie}\left(R_{u}(P)\right)$ give rise to a holomorphic vector bundle $E_{P}(\mathfrak{n}):=E_{P} \times{ }^{P} \mathfrak{n}$ on $X$. Then we have the following (see Theorem 3.3.2).

Theorem 1.1.2. Suppose that $H^{1}\left(X, E_{P}(\mathfrak{n}) \otimes \Omega_{X}^{1}(\log D)\right)=0$. Then $E_{P}$ admits a logarithmic connection singular along $D$ if $E_{L}$ admits a logarithmic connection singular along $D$.

In Section $\S 4$, we discuss how existence of logarithmic connection on $E_{G}$ singular along $D$ can be ensured from existence of logarithmic connection on $\left.E_{G}\right|_{X_{n}}$, where $X_{n}$ is some sufficiently high degree hypersurface in $X$ intersecting $D$ properly. More precisely, we fix an embedding $X \hookrightarrow \mathbb{C P}^{N}$, for some $N>0$. By a hypersurface $X_{n}$ of degree $n$ in $X$, we mean $X \cap H_{n}$, for some hypersurface $H_{n}$ in $\mathbb{C P}^{N}$ of degree $n$. In [Ati57, Proposition 21], it is shown that if $\operatorname{dim}_{\mathbb{C}}(X) \geq 3$, then $E_{G}$ admits a holomorphic connection if and only if for some smooth hypersurface $X_{n}$ in $X$ of sufficiently large degree, the principal $G$-bundle $\left.E_{G}\right|_{X_{n}}$ admits a holomorphic connection. However, it is shown in [Ati57] that this result fails if $\operatorname{dim}_{\mathbb{C}}(X)=2$; see also [BG18]. Also there are no complete answers known for this problem if $\operatorname{dim}_{\mathbb{C}}(X)=2$. We prove the following analogue of [Ati57, Proposition 21] in the case of logarithmic connections on $E_{G}$ singular along $D$ in $X$ (see Theorem 4.1.1). 
Theorem 1.1.3. With the above notations, if $\operatorname{dim}_{\mathbb{C}}(X) \geq 3$ and $D \subset X$ a reduced effective divisor in $X$, then $E_{G}$ admits a logarithmic connection singular along $D$ if and only if for some smooth hypersurface $X_{n}$ in $X$ of sufficiently large degree $n$, intersecting D properly, the principal G-bundle $\left.E_{G}\right|_{X_{n}}$ on $X_{n}$ admits a logarithmic connection singular along $D \cap X_{n}$.

\section{PRELIMINARIES}

2.1. Simple normal crossing divisor. Let $X$ be a connected smooth complex projective variety of dimension at least one. We denote by $T X$ (respectively, $\Omega_{X}^{1}$ ) the tangent bundle (respectively, cotangent bundle) of $X$. The ideal sheaf $\mathscr{I}_{D}$ of an effective divisor $D$ on $X$ is a line bundle on $X$, denoted $\mathcal{O}_{X}(-D)$.

Definition 2.1.1. An effective divisor $D$ on $X$ is said to be a simple normal crossing divisor if $D$ is reduced, each irreducible components of $D$ are smooth, and for each point $x \in X$, there is a system of regular elements (local parameters) $z_{1}, \ldots, z_{n} \in \mathfrak{m}_{x}$ such that the stalk $\mathcal{O}_{X}(-D)_{x}$ of the line bundle $\mathcal{O}_{X}(-D)$ at $x$ is generated by the product $z_{1} \cdots z_{r}$, for some integer $r$ with $1 \leq r \leq n$.

In other words, a simple normal crossing divisor on $X$ is a reduced effective divisor $D$ on $X$, all of whose irreducible components are smooth, and locally for some choice of coordinate functions $\left(z_{1}, \ldots, z_{n}\right)$ around a point $x_{0} \in U \subset X, D \cap U$ is given by an equation $z_{1} \cdots z_{r}=0$, for some integer $r$ with $1 \leq r \leq n$. This means, the irreducible components of $D$ passing through $x_{0}$ are given by the equations $z_{i}=0$, for $i=1, \ldots, r$, and they intersects each others transversally.

2.2. Logarithmic connection. Let $D \subset X$ be a reduced effective divisor on $X$. For an integer $p \geq 0$, a meromorphic $p$-form on $X$ is a section of $\Omega_{X}^{p}(D):=\Omega_{X}^{p} \otimes_{\mathcal{O}_{X}} \mathcal{O}_{X}(D)$. A meromorphic $p$-form $\alpha \in\left(\Omega_{X}^{p}(D)\right)(U)$ on an open subset $U \subset X$ is said to have a logarithmic pole along $D$ if $\alpha$ is holomorphic on $U \backslash(U \cap D)$ and $\alpha$ has pole of order at most one along each irreducible component of $D$, and the same holds for $d \alpha$, where $d$ denotes the holomorphic exterior differential operator (see [Voi07, p. 197]). Let $\Omega_{X}^{p}(\log D)$ be the subsheaf of meromorphic $p$-forms on $X$ with at most logarithmic pole along $D$.

Let $p: E \rightarrow X$ be a holomorphic vector bundle of rank $r$ on $X$. By abuse of notation, we denote by $E$ the sheaf of holomorphic sections of $p: E \rightarrow X$; this is a locally free coherent sheaf of $\mathcal{O}_{X}$-modules of rank $r$ on $X$.

Definition 2.2.1. A logarithmic connection on $E$ singular along $D$ is a $\mathbb{C}$-linear sheaf homomorphism

$$
\nabla: E \longrightarrow E \otimes_{\mathcal{O}_{X}} \Omega_{X}^{1}(\log D)
$$


satisfying the Leibniz rule

$$
\nabla(f \cdot s)=f \nabla(s)+s \otimes d f,
$$

for all locally defined section $f$ of $\mathcal{O}_{X}$ and locally defined section $s$ of $E$.

2.3. Residue of a logarithmic connection. We now recall the definition of reside of a logarithmic connection from [Del70, Oht82]. Let $D$ be a simple normal crossing divisor on $X$. Write $D=\bigcup_{j \in J} D_{j}$ as a union of all of its irreducible components. Let $E$ be a holomorphic vector bundle of rank $r$ on $X$ admitting a logarithmic connection

$$
\nabla: E \longrightarrow E \otimes_{\mathcal{O}_{X}} \Omega_{X}^{1}(\log D)
$$

singular along $D$. Since each irreducible component $D_{j}$ of $D$ are smooth, using Poincaré residue map (see [Voi07, p. 211], [GH94, p. 147]), we have the following homomorphism

$$
\operatorname{Res}_{D_{j}}: E \otimes \Omega_{X}^{1}(\log D) \longrightarrow E \otimes \mathcal{O}_{D_{j}}, \forall j
$$

Then the composite map

$$
\operatorname{Res}_{D_{j}} \circ \nabla:\left.\left.E\right|_{D_{j}} \longrightarrow E\right|_{D_{j}}
$$

is a $\mathcal{O}_{D_{j}}$-module homomorphism, and hence defines a section

$$
\operatorname{Res}_{D_{j}}(\nabla) \in H^{0}\left(D_{j},\left.\mathcal{E} n d(E)\right|_{D_{j}}\right)
$$

called the residue of $\nabla$ along $D_{j}$. For the sake of completeness, we recall explicit description of the reside of $\nabla$ along $D_{j}$ using local coordinates; [Oht82].

Since $D$ is a simple normal crossing divisor on $X$, we can choose an open cover $\left\{U_{\lambda}: \lambda \in\right.$ $\Lambda$ \} of $X$ such that for each $\lambda \in \Lambda$,

(I) $\left.E\right|_{U_{\lambda}}$ is trivial, and

(II) for each irreducible component $D_{j}$ of $D$, with $D_{j} \cap U_{\lambda} \neq \emptyset$, we can choose a local coordinate function $f_{\lambda j} \in \mathcal{O}_{X}\left(U_{\lambda}\right)$ for a local coordinate system on $U_{\lambda}$, such that $f_{\lambda j}$ is a defining equation of $D_{j} \cap U_{\lambda}$. If $D_{j} \cap U_{\lambda}=\emptyset$, we take $f_{\lambda j}=1$.

If $\nabla_{\lambda}$ is the connection matrix of $\nabla$ with respect to a holomorphic local frame $s_{\lambda}=$ $\left(s_{\lambda 1}, \ldots, s_{\lambda r}\right)$ for $E$ on $U_{\lambda}$, then we have

$$
\nabla\left(s_{\lambda}\right)=\nabla_{\lambda} \otimes s_{\lambda}
$$

where $\nabla_{\lambda}$ is a $r \times r$ matrix whose entries are holomorphic sections of $\Omega_{X}^{1}(\log D)$ over $U_{\lambda}$. For each $D_{j}$, the matrix $\nabla_{\lambda}$ can be written as

$$
\nabla_{\lambda}=R_{\lambda j} \frac{d f_{\lambda j}}{f_{\lambda j}}+S_{\lambda j}
$$


where $R_{\lambda j}$ is a $r \times r$ matrix with entries in $\mathcal{O}_{X}\left(U_{\lambda}\right)$ and $S_{\lambda j}$ is a $r \times r$ matrix with entries in $\left(\Omega_{X}^{1}(\log D)\right)\left(U_{\lambda}\right)$ with simple pole along $\bigcup_{j^{\prime} \neq j} D_{j^{\prime}}$. Then

$$
\operatorname{Res}_{D_{j}}\left(\nabla_{\lambda}\right):=\left.R_{\lambda j}\right|_{U_{\lambda} \cap D_{j}}
$$

is a $r \times r$ matrix whose entries are holomorphic functions on $U_{\lambda} \cap D_{j}$; it is independent of choice of local defining equation $f_{\lambda j}$ for $D_{j}$. Then $\left\{\operatorname{Res}_{D_{j}}\left(\nabla_{\lambda}\right)\right\}_{\lambda \in \Lambda}$ defines a holomorphic global section

$$
\operatorname{Res}_{D_{j}}(\nabla) \in H^{0}\left(D_{j}, \mathcal{E} n d\left(\left.E\right|_{D_{j}}\right)\right)
$$

known as the residue of $\nabla$ along $D_{j}$.

Remark 2.3.1. If we further assume that intersections of any finite number of irreducible components of $D$ are connected, then the Chern classes of $E$ can be computed in terms of the residues of the logarithmic connection $\nabla$ along the irreducible components of $D$, and the first Chern classes of the line bundles associated to the irreducible components of $D$; see [Oht82, Theorem 3, p. 16].

\section{Logarithmic Connection On Principal Bundles}

3.1. Logarithmic Atiyah exact sequence. Let $G$ be a connected complex Lie group with Lie algebra g. Let

$$
p: E_{G} \longrightarrow X
$$

be a holomorphic principal $G$-bundle on $X$. The holomorphic $G$-action on $E_{G}$ induces a holomorphic $G$-action on the holomorphic tangent bundle $T E_{G}$ of $E_{G}$, and the associated quotient $\operatorname{At}\left(E_{G}\right):=T E_{G} / G$ is a holomorphic vector bundle on $X$, known as the Atiyah bundle of $E_{G}$; the sections of $\operatorname{At}\left(E_{G}\right)$ are given by $G$-invariant holomorphic vector fields on $E_{G}$. Let $\operatorname{ad}\left(E_{G}\right):=E_{G} \times{ }^{G} \mathfrak{g}$ be the adjoint vector bundle associated to the adjoint representation of $G$ to its Lie algebra $\mathfrak{g}$. The surjective submersion $p$ in (3.1.1) induces a short exact sequence of holomorphic vector bundles on $X$,

$$
0 \longrightarrow \operatorname{ad}\left(E_{G}\right) \longrightarrow \operatorname{At}\left(E_{G}\right) \stackrel{d^{\prime} p}{\longrightarrow} T X \longrightarrow 0,
$$

called the Atiyah exact sequence of $E_{G}$. A holomorphic connection on $E_{G}$ is given by a holomorphic vector bundle homomorphism $\eta: T X \rightarrow \operatorname{At}\left(E_{G}\right)$ such that $d^{\prime} p \circ \eta=\operatorname{Id}_{T X}$; see [Ati57]. We now modify the exact sequence (3.1.2) to define a logarithmic Atiyah exact sequence.

Let $D$ be a reduced effective divisor on $X$. Then $T X(-\log D):=\left(\Omega_{X}^{1}(\log D)\right)^{\vee}$ is a locally free $\mathcal{O}_{X}$-submodule of $T X$. In fact, we have $T X(-D) \subseteq T X(-\log D) \subset T X$. Then we have a locally free $\mathcal{O}_{X}$-submodule $\mathcal{A}_{D}\left(E_{G}\right):=\left(d^{\prime} p\right)^{-1}(T X(-\log D))$ of $\operatorname{At}\left(E_{G}\right)$ which fits into the following short exact sequence of locally free $\mathcal{O}_{X}$-modules

$$
0 \longrightarrow \operatorname{ad}\left(E_{G}\right) \stackrel{\iota_{D}}{\longrightarrow} \mathcal{A}_{D}\left(E_{G}\right) \stackrel{\widetilde{d^{\prime} p}}{\longrightarrow} T X(-\log D) \longrightarrow 0
$$


called the logarithmic Atiyah exact sequence of $E_{G}$ for the divisor $D$, (see also [BDP18]). Moreover, we have the following commutative diagram of $\mathcal{O}_{X}$-module homomorphisms

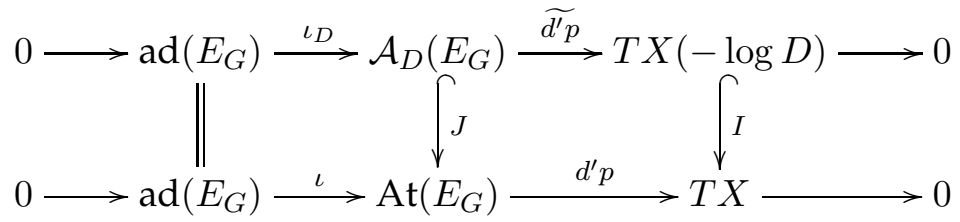

Let $E$ be a holomorphic vector bundle $E$ of rank $n$ on $X$. Let $p: E_{\mathrm{GL}_{n}(\mathbb{C})} \longrightarrow X$ be the holomorphic frame bundle of $E$; this is a principal $\mathrm{GL}_{n}(\mathbb{C})$-bundle on $X$. Note that, $\operatorname{ad}\left(E_{\mathrm{GL}_{n}(\mathbb{C})}\right)$ is naturally isomorphic to $\mathcal{E}$ d $(E)$.

Proposition 3.1.1. $E$ admits a logarithmic connection $\nabla: E \rightarrow E \otimes \Omega_{X}^{1}(\log D)$ singular along $D$ if and only if the exact sequence in (3.1.3) associated to $E_{\mathrm{GL}_{n}(\mathbb{C})}$ splits holomorphically.

Proof. Let $G=\mathrm{GL}_{n}(\mathbb{C})$. Let $\operatorname{Der}_{\mathbb{C}}\left(E_{G}\right)$ be the sheaf of $\mathbb{C}$-linear derivations of $\mathcal{O}_{E_{G}}$. Then there is a natural $\mathcal{O}_{E_{G}}$-module isomorphism $\operatorname{Der} \mathbb{C}\left(E_{G}\right) \stackrel{\widetilde{\sim}}{\rightarrow} \mathcal{H o m}\left(\Omega_{E_{G}}^{1}, \mathcal{O}_{E_{G}}\right)=T E_{G}$ defined by sending a locally defined $\mathbb{C}$-linear derivation $\xi$ of $\mathcal{O}_{E_{G}}$ to the unique $\mathcal{O}_{E_{G}}$-module homomorphism $\widetilde{\xi}: \Omega_{E_{G}}^{1} \rightarrow \mathcal{O}_{E_{G}}$ such that $\widetilde{\xi} \circ d=\xi$, where $d: \mathcal{O}_{E_{G}} \rightarrow \Omega_{E_{G}}^{1}$ is the Kähler differential operator on $E_{G}$. Then the $G$-invariant sections of $\operatorname{Der}_{\mathbb{C}}\left(E_{G}\right)$ descend to sections of $\operatorname{At}\left(E_{G}\right)$.

Now it is clear that given a $\mathcal{O}_{X}$-module homomorphism $\eta: T X(-\log D) \rightarrow \mathcal{A}_{D}(E)$ with $\eta \circ \widetilde{d^{\prime} p}=\operatorname{Id}_{T X(-\log D)}$, for each locally defined section $\xi$ of $T X(-\log D)$, its image $\eta(\xi)$ defines a $G$-invariant $\mathbb{C}$-linear derivation of $E$. Thus we have a logarithmic connection on $E$ singular along $D$. Conversely, given a logarithmic connection $\nabla: E \rightarrow E \otimes \Omega_{X}^{1}(\log D)$ singular along $D$, for each locally defined section $\xi$ of $T X(-\log D)=\mathcal{H o m}\left(\Omega_{X}^{1}(\log D), \mathcal{O}_{X}\right)$, we get a $\mathrm{GL}_{n}(\mathbb{C})$-invariant $\mathbb{C}$-linear derivation $\nabla_{\xi}:=\left(\mathrm{Id}_{E} \otimes \xi\right) \circ \nabla$ of $E$. This defines a splitting of the short exact sequence (3.1.3).

The above Proposition 3.1.1 motivates us to define the following (see also [BDPS17, §2.2]).

Definition 3.1.2. Let $p: E_{G} \rightarrow X$ be a holomorphic principal $G$-bundle on $X$. A $\log a-$ rithmic connection on $E_{G}$ singular along $D$ is a holomorphic vector bundle homomorphism $\eta: T X(-\log D) \rightarrow \mathcal{A}_{D}\left(E_{G}\right)$ such that $\widetilde{d^{\prime} p} \circ \eta=\operatorname{Id}_{T X(-\log D)}$, where $\widetilde{d^{\prime} p}$ is the homomorphism in (3.1.3).

We refer the exact sequence (3.1.3) as the logarithmic Atiyah exact sequence of $E_{G}$ associated to the divisor $D$. The exact sequence (3.1.3) defines a cohomology class

$$
\Phi_{D}(E) \in H^{1}\left(X, \operatorname{ad}\left(E_{G}\right) \otimes \Omega_{X}^{1}(\log D)\right),
$$


which we call the logarithmic Atiyah class of $E$ along $D$, such that the exact sequence (3.1.3) splits holomorphically if and only if $\Phi_{D}(E)=0$.

3.2. Residue of logarithmic connection on a principal bundle. Let $D$ be a simple normal crossing divisor on $X$, locally defined by $z_{1} \cdots z_{r}=0$. Let us denote by $D_{j}$ the irreducible component of $D$ locally defined by $z_{j}=0$, for each $j=1, \ldots, r$. Let $T X(-\log D)$ be the dual of $\Omega_{X}^{1}(\log D)$; this is a locally free coherent sheaf of $\mathcal{O}_{X}$-modules of rank $d=\operatorname{dim}_{\mathbb{C}}(X)$, with local frame fields given by $\left(z_{1} \frac{\partial}{\partial z_{1}}, \ldots, z_{r} \frac{\partial}{\partial z_{r}}, \frac{\partial}{\partial z_{r+1}}, \ldots, \frac{\partial}{\partial z_{d}}\right)$. For each $j=1, \ldots, r$, over $D_{j}$, we can identify $z_{j} \frac{\partial}{\partial z_{j}}$ with 1 ; this identification is independent of choice of local coordinate system $\left(z_{1}, \ldots, z_{d}\right)$ on $X$ such that $D_{i}$ is locally given by vanishing locus of $z_{i}$, for all $i=1, \ldots, r$. Thus, $\left.T X(-\log D)\right|_{D_{j}}$ is locally free $\mathcal{O}_{D_{j}}$-module generated by

$$
\left(z_{1} \frac{\partial}{\partial z_{1}}, \ldots, z_{j-1} \frac{\partial}{\partial z_{j-1}}, 1, z_{j+1} \frac{\partial}{\partial z_{j+1}}, \ldots, z_{r} \frac{\partial}{\partial z_{r}}, \frac{\partial}{\partial z_{r+1}}, \ldots, \frac{\partial}{\partial z_{d}}\right) .
$$

Therefore, we have an injective homomorphism $\left.\mathcal{O}_{D_{j}} \longrightarrow T X(-\log D)\right|_{D_{j}}$. Let

$$
\eta: T X(-\log D) \longrightarrow \mathcal{A}_{D}\left(E_{G}\right)
$$

be a logarithmic connection on $E_{G}$ singular along $D$; that means, $\eta$ is an $\mathcal{O}_{X}$-module homomorphism such that $\widetilde{d^{\prime} p} \circ \eta=\operatorname{Id}_{T X(-\log D)}$ (see (3.1.3)). Note that the image of $\left.\eta\right|_{\mathcal{O}_{D_{j}}}$ lands inside $\left.\left.\operatorname{ad}\left(E_{G}\right)\right|_{D_{j}} \subset \mathcal{A}_{D_{j}}\left(E_{G}\right)\right|_{D_{j}}$. This gives a section

$$
\operatorname{Res}_{D_{j}}(\eta) \in H^{0}\left(D_{j},\left.\operatorname{ad}\left(E_{G}\right)\right|_{D_{j}}\right),
$$

called the residue of $\eta$ along $D_{j}$, for all $j=1, \ldots, r$. Then we have the following.

Proposition 3.2.1. Let $E$ be a holomorphic vector bundle of rank $n$ on $X$, and let $E_{\mathrm{GL}_{n}(\mathbb{C})}$ be the holomorphic frame bundle of $E$. If $\eta$ in (3.2.1) is the logarithmic connection on $E_{\mathrm{GL}_{n}(\mathbb{C})}$ associated to a logarithmic connection $\nabla$ on $E$ as defined in (2.2.1), then for each irreducible component $D_{j}$ of $D$, we have

$$
\operatorname{Res}_{D_{j}}(\nabla)=\operatorname{Res}_{D_{j}}(\eta)
$$

where $\operatorname{Res}_{D_{j}}(\nabla)$ is as defined in (2.3.5) and $\operatorname{Res}_{D_{j}}(\eta)$ is as defined in (3.2.2).

Proof. Follows from the proof of Proposition 3.1.1 and the definition of residue in (2.3.1).

3.3. Extension of structure group. Let $G$ and $H$ be two connected complex Lie groups with Lie algebras $\mathfrak{g}$ and $\mathfrak{h}$, respectively. Let $f: H \longrightarrow G$ be a homomorphism of complex Lie groups, and $d f: \mathfrak{h} \longrightarrow \mathfrak{g}$ the Lie algebra homomorphism induced by $f$. Let $p: E_{H} \rightarrow X$ be a holomorphic principal $H$-bundle on $X$. Then we have a holomorphic principal $G$-bundle $p^{\prime}: E_{G}:=E_{H}(G) \rightarrow X$ on $X$ obtained by extending the structure group of $E_{H}$ by the homomorphism $f$. Then there is a natural vector bundle homomorphisms $\alpha: \operatorname{ad}\left(E_{H}\right) \longrightarrow$ 
$\operatorname{ad}\left(E_{G}\right)$ and $\beta: \operatorname{At}\left(E_{H}\right) \longrightarrow \operatorname{At}\left(E_{G}\right)$ induced by $f$. Then we have the following commutative diagram of vector bundle homomorphisms with two rows exact (see [Ati57]).

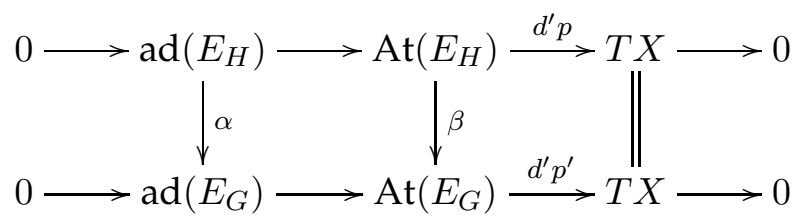

Let $D$ be a reduced effective divisor on $X$. Then the commutative diagram (3.1.4) and (3.3.1) gives the following commutative diagram of vector bundle homomorphisms with two rows exact.

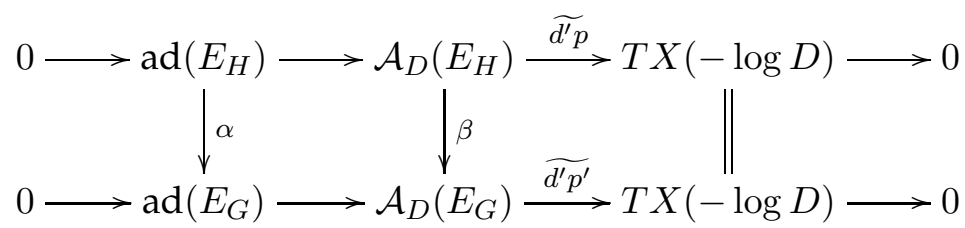

If $\eta: T X(-\log D) \rightarrow \mathcal{A}_{D}\left(E_{H}\right)$ is a holomorphic vector bundle homomorphism with $\widetilde{d^{\prime} p} \cap \eta=$ $\operatorname{Id}_{T X(-\log D)}$, then $f_{*}(\eta):=\beta \circ \eta$ satisfies $\widetilde{d^{\prime} p^{\prime}} \circ\left(f_{*} \eta\right)=\operatorname{Id}_{T X(-\log D)}$. Consequently, if $D$ is a simple normal crossing divisor $D$ in $X$, for each irreducible component $D_{j}$ of $D$, we have $\operatorname{Res}_{D_{j}}\left(f_{*} \eta\right)=\alpha \circ \operatorname{Res}_{D_{j}}(\eta)$; (see also [BDPS17, §2.4]).

In fact, it follows from commutativity of the diagram (3.3.2) that there is a natural homomorphism of cohomologies

$$
f_{*}: H^{1}\left(X, \operatorname{ad}\left(E_{H}\right) \otimes \Omega_{X}^{1}(\log D)\right) \longrightarrow H^{1}\left(X, \operatorname{ad}\left(E_{G}\right) \otimes \Omega_{X}^{1}(\log D)\right),
$$

induced by $f$, which sends the cohomology class $\Phi_{D}\left(E_{H}\right)$ to $\Phi_{D}\left(E_{G}\right)$; see (3.1.5). Since the homomorphism (3.3.3) is not necessarily injective, in general, existence of a logarithmic connection on $E_{G}$ singular along $D$ may not ensure existence of a logarithmic connection on $E_{H}$ singular along $D$. However, if $f: H \longrightarrow G$ is an injective homomorphism of connected affine algebraic groups over $\mathbb{C}$ with $H$ reductive, then the above homomorphism (3.3.3) can be shown to be injective (see the proof of [BDPS17, Lemma 3.3] for more details). Therefore, from the above discussions, we have the following.

Proposition 3.3.1. With the above notations, $E_{G}$ admits a logarithmic connection singular along $D$ if $E_{H}$ admits a logarithmic connection singular along $D$. Converse holds if $f: H \rightarrow G$ is an injective homomorphism of connected affine algebraic groups over $\mathbb{C}$ with $H$ reductive.

Let $G$ be a connected reductive affine algebraic group over $\mathbb{C}$. Let $P$ be a parabolic subgroup of $G$. Let $R_{u}(P)$ be the unipotent radical of $P$. Then there is a closed connected algebraic subgroup $L \subset P$ such that the restriction to $L$ of the quotient homomorphism

$$
q: P \longrightarrow P / R_{u}(P),
$$


is an isomorphism of algebraic groups over $\mathbb{C}$. Clearly, $L$ is reductive; and it is known as the Levi factor of $P$ (see e.g., [Mil17, p. 559]). Consider the homomorphism

$$
q^{\prime}:=\left(\left.q\right|_{L}\right)^{-1} \circ q: P \longrightarrow L .
$$

Let $E_{P}$ be a homomorphic principal $P$-bundle on $X$. Let $E_{L}:=E_{P}(L)$ be the holomorphic principal $L$-bundle on $X$ obtained by extending the structure group of $E_{P}$ by the homomorphism $q^{\prime}$ in (3.3.4). The Lie algebra $\mathfrak{n}:=\operatorname{Lie}\left(R_{u}(P)\right)$ of $R_{u}(P)$ is the nilpotent radical of the Lie algebra $\mathfrak{p}:=\operatorname{Lie}(P)$ of $P$. The action of $P$ on $\mathfrak{n}$ gives rise to a holomorphic vector bundle $E_{P}(\mathfrak{n}):=E_{P} \times{ }^{P} \mathfrak{n}$ on $X$. Note that, $E_{P}(\mathfrak{n})$ is a subbundle of $\operatorname{ad}\left(E_{P}\right)=E_{P}(\mathfrak{p})$, and the associated quotient vector bundle $\operatorname{ad}\left(E_{P}\right) / E_{P}(\mathfrak{n})$ is isomorphic to $E_{P}(\mathfrak{l}) \cong \operatorname{ad}\left(E_{L}\right)$, where $\mathfrak{l}=\operatorname{Lie}(L)$. Then we have the following.

Theorem 3.3.2. With the above notations, if $H^{1}\left(X, E_{P}(\mathfrak{n}) \otimes \Omega_{X}^{1}(\log D)\right)=0$, then $E_{P}$ admits a logarithmic connection singular along $D$ whenever $E_{L}$ admits a logarithmic connection singular along $D$.

Proof. Replacing $H$ by $P$ and $G$ by $L$ in the commutative diagram (3.3.2), we have the following commutative diagram of holomorphic vector bundle homomorphisms, with all rows and columns exact.

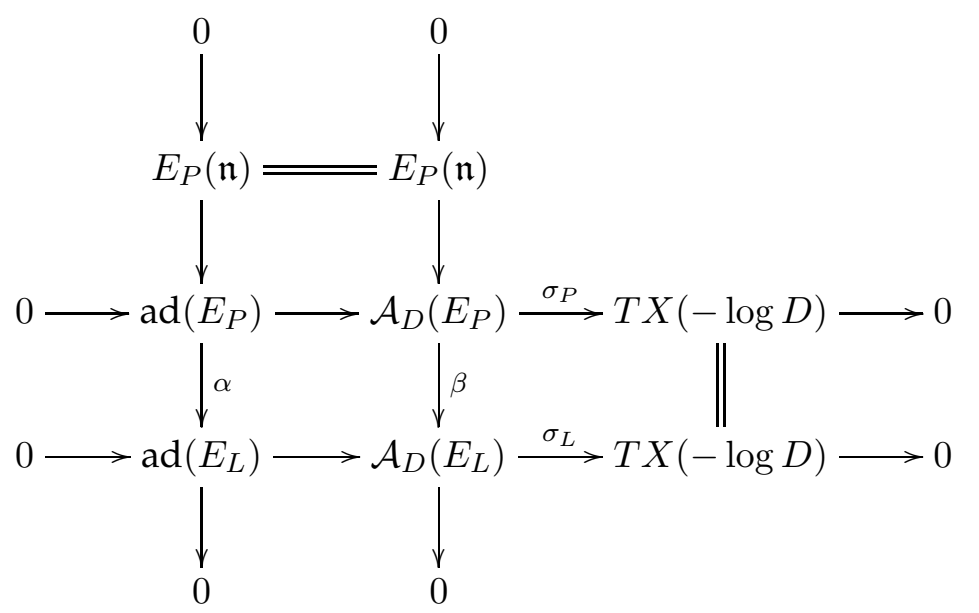

Let $\eta: T X(-\log D) \rightarrow \mathcal{A}_{D}\left(E_{L}\right)$ be an $\mathcal{O}_{X}$-module homomorphism such that $\sigma_{L} \circ \eta=$ $\operatorname{Id}_{T X(-\log D)}$, where $\sigma_{L}$ is the homomorphism in (3.3.5). Let $\mathcal{F}:=\beta^{-1}(\eta(T X(-\log D))) \subset$ $\mathcal{A}_{D}\left(E_{P}\right)$. This fits into the following short exact sequence of $\mathcal{O}_{X}$-modules

$$
0 \longrightarrow E_{P}(\mathfrak{n}) \longrightarrow \mathcal{F} \longrightarrow T X(-\log D) \longrightarrow 0 .
$$

Then the logarithmic Atiyah exact sequence for $E_{P}$ in (3.3.5) splits $\mathcal{O}_{X}$-linearly if the exact sequence (3.3.6) splits $\mathcal{O}_{X}$-linearly. Since the obstruction for splitting of the exact sequence (3.3.6) lies in $H^{1}\left(X, E_{P}(\mathfrak{n}) \otimes \Omega_{X}^{1}(\log D)\right)$, the result follows. 


\section{EXISTENCE OF LOGARITHMIC CONNECTION}

4.1. Restriction theorem for logarithmic connection. Let $X$ be a smooth complex projective variety of dimension $d \geq 1$. Fix an embedding of $X$ into a complex projective space $\mathbb{C P}^{N}$, for some positive integer $N$. A hypersurface $X_{n}$ of degree $n$ in $X$ is given by $X \cap H_{n}$, where $H_{n}$ is a hypersurface of degree $n$ in $\mathbb{C P}^{N}$. For general hypersurfaces $H_{n}$, we get $X_{n}=X \cap H_{n}$ smooth [Har77]. Let $\operatorname{Div}(X)$ be the group of all divisors in $X$. For $D_{1}, D_{2} \in \operatorname{Div}(X)$, we say that $D_{1}$ and $D_{2}$ meets properly if for each prime divisor $V$ (respectively, $W$ ) appearing with non-zero coefficient in $D_{1}$ (respectively, $D_{2}$ ), we have $\operatorname{dim}(V \cap W)=d-2$. It is clear that if two reduced effective divisors $D_{1}, D_{2} \in \operatorname{Div}(X)$ meets properly, then $D_{1} \cap D_{2}$ is a divisor in both $D_{1}$ and $D_{2}$.

Let $G$ be a connected complex Lie group, and $E_{G}$ a holomorphic principal $G$-bundle on $X$. Then we have the following result.

Theorem 4.1.1. Assume that $\operatorname{dim}_{\mathbb{C}}(X) \geq 3$ and $D \subset X$ a reduced effective divisor in $X$. Then $E_{G}$ admits a logarithmic connection singular along $D$ if and only if for some smooth hypersurface $X_{n}$ of sufficiently large degree $n$, which intersects D properly, the principal $G$-bundle $\left.E_{G}\right|_{X_{n}}$ on $X_{n}$ admits a logarithmic connection singular along $D \cap X_{n}$.

Proof. For any divisor $H$ on $X$, we denote by $\mathcal{O}_{X}(H)$ the line bundle on $X$ associated to $H$. Let $\mathcal{F}$ be a coherent sheaf of $\mathcal{O}_{X}$-modules on $X$. Consider the exact sequence of sheaves

$$
0 \longrightarrow \mathcal{F} \otimes \mathcal{O}_{X}\left(-X_{n}\right) \longrightarrow \mathcal{F} \longrightarrow \iota_{n *}\left(\left.\mathcal{F}\right|_{X_{n}}\right) \longrightarrow 0
$$

where $\iota_{n}: X_{n} \hookrightarrow X$ is the inclusion morphism. Since $\operatorname{dim}_{\mathbb{C}}(X) \geq 3$, it follows from Serre's theorem [Har77, p. 228] that for $n \gg 0$, we have

$$
H^{i}\left(X, \mathcal{F} \otimes \mathcal{O}_{X}\left(-X_{n}\right)\right)=0, \quad \forall i=1,2 .
$$

Then the long exact sequence of cohomologies associated to the short exact sequence (4.1.1) gives an isomorphism.

$$
H^{1}(X, \mathcal{F}) \stackrel{\cong}{\longrightarrow} H^{1}\left(X_{n},\left.\mathcal{F}\right|_{X_{n}}\right)
$$

Since $X_{n}$ intersects $D$ properly by assumption, $D_{n}:=X_{n} \cap D$ is an effective divisor in $X_{n}$, and we have a natural isomorphism $\left.\mathcal{O}_{X}(D)\right|_{X_{n}} \cong \mathcal{O}_{X_{n}}\left(D_{n}\right)$. Then from [Har77, Chapter II, Theorem 8.17], we have an exact sequence of $\mathcal{O}_{X_{n}}$-modules

$$
\left.0 \longrightarrow\left(\mathscr{I}_{X_{n}} / \mathscr{I}_{X_{n}}^{2}\right) \otimes \mathcal{O}_{X_{n}}\left(D_{n}\right) \longrightarrow \Omega_{X}^{1}(D)\right|_{X_{n}} \stackrel{\xi}{\longrightarrow} \Omega_{X_{n}}^{1}\left(D_{n}\right) \longrightarrow 0,
$$

where $\mathscr{I}_{X_{n}}$ is the ideal sheaf of the hypersurface $X_{n}$ in $X$. Note that there is a natural $\mathcal{O}_{X_{n}}{ }^{-}$ module isomorphism

$$
\left.\Omega_{X}^{1}(\log D)\right|_{X_{n}} \stackrel{\cong}{\longrightarrow} \xi^{-1}\left(\Omega_{X_{n}}^{1}\left(\log D_{n}\right)\right)
$$


Since $\mathscr{I}_{X_{n}} /\left.\mathscr{I}_{X_{n}}^{2} \cong \mathcal{O}_{X}\left(-X_{n}\right)\right|_{X_{n}}$, from (4.1.4) using (4.1.5) we have the following short exact sequence of $\mathcal{O}_{X_{n}}$-modules

$$
\left.\left.0 \longrightarrow \mathcal{O}_{X}\left(D-X_{n}\right)\right|_{X_{n}} \longrightarrow \Omega_{X}^{1}(\log D)\right|_{X_{n}} \longrightarrow \Omega_{X_{n}}^{1}\left(\log D_{n}\right) \longrightarrow 0 .
$$

Now tensoring the exact sequence (4.1.6) with $\left.\operatorname{ad}\left(E_{G}\right)\right|_{X_{n}}$, we get the following short exact sequence of $\mathcal{O}_{X_{n}}$-modules

$$
\begin{array}{r}
\left.\left.0 \longrightarrow\left(\operatorname{ad}\left(E_{G}\right) \otimes \mathcal{O}_{X}\left(D-X_{n}\right)\right)\right|_{X_{n}} \longrightarrow\left(\operatorname{ad}\left(E_{G}\right) \otimes \Omega_{X}^{1}(\log D)\right)\right|_{X_{n}} \\
\left.\longrightarrow \operatorname{ad}\left(E_{G}\right)\right|_{X_{n}} \otimes \Omega_{X_{n}}^{1}\left(\log D_{n}\right) \longrightarrow 0 .
\end{array}
$$

Now taking $\mathcal{F}=\operatorname{ad}\left(E_{G}\right) \otimes \mathcal{O}_{X}(D)$ and $\mathcal{F}=\operatorname{ad}\left(E_{G}\right) \otimes \mathcal{O}_{X}\left(D-X_{n}\right)$ in (4.1.2), we get

$$
H^{1}\left(X, \operatorname{ad}\left(E_{G}\right) \otimes \mathcal{O}_{X}\left(D-X_{n}\right)\right)=0=H^{2}\left(X, \operatorname{ad}\left(E_{G}\right) \otimes \mathcal{O}_{X}\left(D-2 X_{n}\right)\right),
$$

for $n$ large enough. Fix one such $n \gg 0$. Then applying (4.1.3) for $\mathcal{F}=\operatorname{ad}\left(E_{G}\right) \otimes \mathcal{O}_{X}\left(D-X_{n}\right)$, using (4.1.8) we get

$$
H^{1}\left(X_{n},\left.\left(\operatorname{ad}\left(E_{G}\right) \otimes \mathcal{O}_{X}\left(D-X_{n}\right)\right)\right|_{X_{n}}\right)=0
$$

Now from the long exact sequence of cohomologies associated to (4.1.7), using (4.1.9) we get an exact sequence of cohomologies

$$
0 \longrightarrow H^{1}\left(X_{n},\left.\left(\operatorname{ad}\left(E_{G}\right) \otimes \Omega_{X}^{1}(\log D)\right)\right|_{X_{n}}\right) \longrightarrow H^{1}\left(X_{n}, \operatorname{ad}\left(\left.E_{G}\right|_{X_{n}}\right) \otimes \Omega_{X_{n}}^{1}\left(\log D_{n}\right)\right) .
$$

Now taking $\mathcal{F}=\operatorname{ad}\left(E_{G}\right) \otimes \Omega_{X}^{1}(\log D)$ in (4.1.3), from (4.1.10) we see that the inclusion map $\iota_{n}: X_{n} \hookrightarrow X$ induces an injective homomorphism

$$
\widetilde{\iota_{n}}: H^{1}\left(X, \operatorname{ad}\left(E_{G}\right) \otimes \Omega_{X}^{1}(\log D)\right) \longrightarrow H^{1}\left(X_{n}, \operatorname{ad}\left(\left.E_{G}\right|_{X_{n}}\right) \otimes \Omega_{X_{n}}^{1}\left(\log D_{n}\right)\right) .
$$

The inclusion morphism $\iota_{n}: X_{n} \hookrightarrow X$ induces the following commutative diagram of homomorphisms of sheaves of $\mathcal{O}_{X}$-modules on $X$ with two rows exact.

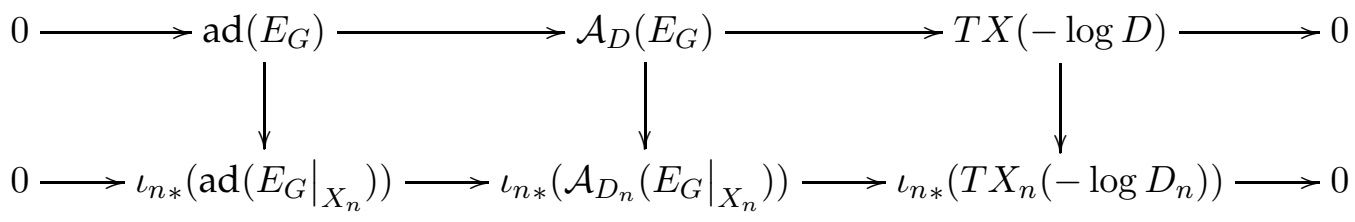

Now one can check that the homomorphism (4.1.11) sends the cohomology class $\Phi_{D}\left(E_{G}\right) \in$ $H^{1}\left(X, \operatorname{ad}\left(E_{G}\right) \otimes \Omega_{X}^{1}(\log D)\right)$, as defined in (3.1.5), to the cohomology class $\Phi_{D_{n}}\left(\left.E_{G}\right|_{X_{n}}\right)$. Thus $\Phi_{D}\left(E_{G}\right)=0$ if and only if $\Phi_{D_{n}}\left(\left.E_{G}\right|_{X_{n}}\right)=0$. This completes the proof.

\section{ACKNOWLEDGEMENTS}

The authors would like to thank Indranil Biswas for suggesting this question. The first named author would like to thank the "Department of Science and Technology" of the Government of India for the INSPIRE fellowship - 15DSTINS009. The second named author would like to thank Arideep Saha for some useful discussions. 


\title{
REFERENCES
}

[AB02] Hassan Azad and Indranil Biswas, On holomorphic principal bundles over a compact Riemann surface admitting a flat connection, Math. Ann., 322, no. 2 (2002), 333-346. doi: 10.1007/s002080100273. [个 1.]

[Ati57] M. F. Atiyah, Complex analytic connections in fibre bundles, Trans. Amer. Math. Soc., 85 (1957), 181-207. doi: 10.2307/1992969. [个 1, 2, 5, and 8.]

[BDP18] Indranil Biswas, Ananyo Dan and Arjun Paul, Criterion for logarithmic connections with prescribed residues, Manuscripta Math., 155, no. 1-2 (2018), 77-88. doi: 10.1007/s00229-017-0935-6. [个 1 and 6.]

[BDPS17] Indranil Biswas, Ananyo Dan, Arjun Paul and Arideep Saha, Logarithmic connections on principal bundles over a Riemann surface, Internat. J. Math., 28, no. 12 (2017), 1750088, 18 pp. doi: 10.1142/S0129167X17500884. [个 1, 6, and 8.]

[BG18] Indranil Biswas and Sudarshan Gurjar, Connections and restrictions to curves, C. R. Math. Acad. Sci. Paris 356 (2018), no. 6, 674-678. doi: 10.1016/j.crma.2018.05.004. [个 2.]

[Del70] Pierre Deligne, Équations différentielles à points singuliers réguliers, Lecture Notes in Mathematics, Vol. 163, Springer-Verlag, Berlin-New York (1970). doi: 10.1007/BFb0061194. [个 4.]

[GH94] Phillip Griffiths and Joseph Harris, Principles of algebraic geometry, Wiley Classics Library, John Wiley \& Sons, Inc., New York (1994). doi: 10.1002/9781118032527. [个 4.]

[Har77] Robin Hartshorne, Algebraic geometry, Graduate Texts in Mathematics, No. 52. Springer-Verlag, New York-Heidelberg, 1977. doi: 10.1007/978-1-4757-3849-0. [个 10.]

[Mil17] J. S. Milne, Algebraic groups, The theory of group schemes of finite type over a field, Cambridge Studies in Advanced Mathematics, 170. Cambridge University Press, Cambridge, 2017. doi: 10.1017/9781316711736. [个9.]

[Oht82] Makoto Ohtsuki, A residue formula for Chern classes associated with logarithmic connections, Tokyo J. Math., 5, no. 1 (1982), 13-21. doi: 10.3836/tjm/1270215030. [个 4 and 5.]

[Voi07] Claire Voisin, Hodge theory and complex algebraic geometry. I, Cambridge Studies in Advanced Mathematics, volume 76, Cambridge University Press, Cambridge, english edition (2007). doi: 10.1017/CBO9780511615344. [个 3 and 4.]

[Wei38] André Weil, Gnralisation des fonctions abliennes, J. Math. Pures Appl., 17 (1938), 47-87. [个 1.]

\author{
SUDARSHAN GURJAR \\ DEPARTMENT OF MATHEMATICS, \\ INDIAN INSTITUTE OF TECHNOLOGY BOMBAY, \\ POWAi, MuMBAi 400076, MAHARASHTRA, INDiA. \\ EMAIL: sgurjaremath.iitb.ac.in \\ ARJUN PAUL \\ DEPARTMENT OF MATHEMATICS, \\ INDIAN INSTITUTE OF TECHNOLOGY BOMBAY, \\ POWAi, Mumbai 400076, MAHARASHTRA, INDiA. \\ EMAIL: arjunp@math.iitb.ac.in
}

